\title{
Axioms for Renormalization in Euclidean Quantum Field Theory
}

\author{
Guido Lang and Andrzej Lesniewski \\ Mathematik, ETH-Zentrum, CH-8092 Zürich, Switzerland
}

\begin{abstract}
A set of axioms which fix Euclidean renormalizations up to a finite renormalization is proposed. There exists a one to one correspondence between Euclidean renormalizations and renormalizations in Minkowski space-time satisfying Hepp's axioms. No restrictions on masses are imposed.
\end{abstract}

\section{Introduction}

In [6] Hepp proposed a general axiomatic framework for renormalization theory in perturbative relativistic quantum field theory. A renormalization is a map which assigns to unrenormalized Feynman amplitudes (which are not, in general, tempered distributions) the corresponding tempered distributions (called renormalized Feynman amplitudes) in such a way that some conditions are satisfied. These conditions are motivated by physical considerations, and they include: Lorentz covariance, unitarity and causality. It is shown that all renormalizations which satisfy the axioms are essentially equivalent: the only arbitrariness is a finite renormalization [6] (in renormalizable theories this arbitrariness is removed by fixing the values of masses and charges). Hepp showed also that the usual renormalization schemes satisfy his axioms.

In this paper we are concerned with renormalization theory in Euclidean space-time. The advantages of the Euclidean approach to field theory are well known: the matters simplify considerably. For the history and modern axiomatic results in this direction see [9]. In the context of renormalization theory this was recognized already by Dyson [1]. In order to avoid the problem of poles in the region of integration, he formally replaced $p^{0}$ by $i p^{0}$ in the $p$-space Feynman amplitudes. Weinberg [11] proved the celebrated power counting theorem giving sufficient conditions for the convergence of Euclidean amplitudes and describing their asymptotic behavior in $p$-space. Zimmermann [13] developed a $p$-space renormalization scheme, proved its convergence, and related it to relativistic Feynman amplitudes. In the context of one-loop approximation to the $\phi^{4}$ theory, renormalized Euclidean amplitudes were also discussed by Williams [12]. 
We propose a set of axioms which should be satisfied by any Euclidean renormalization scheme. We prove generally, without resorting to any particular renormalization scheme, and imposing no restrictions on the values of masses, that a renormalization always exists, and is unique up to a finite renormalization. To convince the reader once more that it is easier to do field theory in Euclidean space-time we prove that the Euclidean counterpart of the analytic renormalization satisfies our axioms. The proof is considerably simpler than the corresponding one in the relativistic approach [6]. We show that there exists a natural one to one correspondence between Euclidean and relativistic renormalizations. The way we establish this correspondence is motivated by [2].

The paper is organized as follows. Section II contains the notations. In Sect. III we formulate the axioms for renormalization in Minkowski and Euclidean spacetime. Sections IV-VI contain the formulation and the proof of our main result: the equivalence theorem. In Sect. VII we prove the existence and uniqueness of Euclidean renormalization. Section VIII contains an example: the Euclidean formulation of the analytic renormalization. In Appendix A we prove a technical lemma. Appendix B summarizes some facts about radially analytic functions.

\section{Unrenormalized Amplitudes}

Let $\Gamma=\Gamma(\mathscr{L}, \mathscr{V})$ be a connected graph $(\mathscr{L}$ denotes the set of its internal lines, $\mathscr{V}$ is the set of its vertices). To avoid inessential notational complications, we shall ignore the dependence of renormalizations on external legs in the following. A subgraph $\gamma$ of $\Gamma$ (in symbols : $\gamma \subset \Gamma$ ) is a set $\mathscr{V}(\gamma) \subset \mathscr{V}$ of vertices and the set $\mathscr{L}(\gamma) \subset$ $\mathscr{L}$ of all lines in $\mathscr{L}$ joining two elements of $\mathscr{V}(\gamma)$. Let $\gamma_{1}, \gamma_{2} \subset \Gamma$ be subgraphs. By $\gamma_{1} \cup \gamma_{2}$ we denote the subgraph with $\mathscr{V}\left(\gamma_{1} \cup \gamma_{2}\right)=\mathscr{V}\left(\gamma_{1}\right) \cup \mathscr{V}\left(\gamma_{2}\right)$, while $\gamma_{1} \cap \gamma_{2}$ is defined as the subgraph for which $\mathscr{V}\left(\gamma_{1} \cap \gamma_{2}\right)=\mathscr{V}\left(\gamma_{1}\right) \cap \mathscr{V}\left(\gamma_{2}\right)$. We write $\gamma=\emptyset$, if $\mathscr{V}(\gamma)=\emptyset$. Let $\mathscr{U}=\left\{\gamma_{1}, \ldots, \gamma_{a}\right\}$ be a family of subgraphs of $\Gamma$ such that $\gamma_{i} \neq \emptyset$ $(i=1, \ldots, a), \quad \gamma_{1} \cup \ldots \cup \gamma_{a}=\Gamma$, and $\gamma_{i} \cap \gamma_{j}=\emptyset(i \neq j)$. We call the pair $(\Gamma, \mathscr{U})$ a generalized graph. A subgraph $\gamma \subset \Gamma$ is compatible with $\mathscr{U}$, if for any $\gamma_{i} \in \mathscr{U}$ either $\gamma_{i} \subset \gamma$ or $\gamma_{i} \cap \gamma=\emptyset$. Given a subgraph $\gamma$ compatible with $\mathscr{U}$ we define $\mathscr{U}_{\gamma}=\left\{\gamma_{i} \in \mathscr{U}: \gamma_{i} \subset \gamma\right\}$.

We label the components of $x \in \mathbb{R}^{4 V}(V=|\mathscr{V}|)$ by the elements of $\mathscr{V}$, i.e. $\underline{x}=\left(x_{v}\right)_{v \in \mathscr{V}}$, where $x_{v}=\left(x_{v}^{0}, \mathbf{x}_{v}\right) \in \mathbb{R}^{4}$. Sometimes, however, we find it convenient to use other notations, e.g. $\underline{x}=\left(x_{1}, \ldots, x_{V}\right)$, or

$$
\underline{x}=\left(x_{1,1}, \ldots, x_{1,\left|\mathscr{V}\left(\gamma_{1}\right)\right|}, \ldots, x_{a,\left|\mathscr{V}\left(\gamma_{a}\right)\right|}\right),
$$

for a given generalized graph $(\Gamma, \mathscr{U})$.

In this paper we consider a theory of a single scalar field. The generalization to fields with arbitrary spin is straightforward, although notationally somewhat involved. With a generalized graph $(\Gamma, \mathscr{U})$ we associate the corresponding (relativistic and Euclidean) amplitudes defined as

$$
\begin{gathered}
\overline{\mathscr{F}}_{(\Gamma, \mathscr{U})}(\underline{x})=\prod_{\gamma_{2} \in \mathscr{U}} \Lambda_{\gamma_{t}}^{R}\left(\underline{x}_{\gamma_{2}}\right) \prod_{\sigma \notin \cup \mathscr{L}\left(\gamma_{i}\right)} \bar{\Delta}_{F}\left(x_{i(\sigma)}-x_{f(\sigma)}\right), \\
\mathscr{E}_{(\Gamma, \mathscr{U})}(\underline{x})=\prod_{\gamma_{i} \in \mathscr{U}} \Lambda_{\gamma_{i}}^{E}\left(\underline{x}_{\gamma_{i}}\right) \prod_{\sigma \notin \cup \mathscr{L}\left(\gamma_{i}\right)} S\left(x_{i(\sigma)}-x_{f(\sigma)}\right),
\end{gathered}
$$


where $\underline{x}_{\gamma_{t}}=\left(x_{v}\right)_{v \in \mathscr{V}\left(\gamma_{t}\right)}, i(\sigma), f(\sigma) \in \mathscr{V}$ denote the vertices connected by $\sigma$. The propagators are defined as

$$
\begin{gathered}
\bar{\Delta}_{F}(x)=i(2 \pi)^{-4} \int d^{4} k e^{-i k x}\left(\mu^{2}-k^{2}+i 0\right)^{-1}, \\
S(x)=(2 \pi)^{-4} \int d^{4} k e^{i k x}\left(\mu^{2}+k^{2}\right)^{-1},
\end{gathered}
$$

(as usual, in the relativistic case $k x=k^{0} x^{0}-\mathbf{k} \cdot \mathbf{x}$, while in the Euclidean case $\left.k x=k^{0} x^{0}+\mathbf{k} \cdot \mathbf{x}\right)$. The $\Lambda^{\prime}$ 's are defined as

$$
\Lambda_{\gamma_{i}}^{R}\left(\underline{x}_{\gamma_{i}}\right)= \begin{cases}1, & \text { if } \gamma_{i} \text { is a single vertex }, \\ D_{\gamma_{2}} \prod_{j=1}^{\left|\mathscr{V}\left(\gamma_{i}\right)\right|-1} \delta\left(x_{i, j+1}-x_{i, j}\right), & \text { otherwise }\end{cases}
$$

where $D_{\gamma_{2}}$ is some Lorentz invariant differential operator with constant coefficients, such that $D_{\gamma_{i}}^{*}=(-1)^{\left|\mathscr{V}\left(\gamma_{1}\right)\right|-1} D_{\gamma_{i}}$ (the star means complex conjugation). $\Lambda_{\gamma_{i}}^{E}\left(\underline{x}_{\gamma_{i}}\right)$ is defined by the same formula, but $D_{\gamma_{i}}$ is now a Euclidean invariant differential operator with constant coefficients, such that $D_{\gamma_{i}}^{*}=D_{\gamma_{i}}$. Given $\gamma \subset \Gamma$ compatible with $\mathscr{U}$, the notation $\left.\overline{\mathscr{F}_{(\gamma,}} \mathscr{U}_{\gamma}\right)\left(\underline{x}_{\gamma}\right)$ [respectively $\left.\mathscr{E}_{\left(\gamma, \mathscr{U}_{\gamma}\right)}\left(\underline{x}_{\gamma}\right)\right]$ is clear.

As they stand (II.1) and (II.2) are purely formal expressions. To give them a precise meaning we introduce some notation. Given an open set $\Omega \subset \mathbb{R}^{4 V}$ we denote by $\mathscr{S}(\Omega)$ the set of all $f \in \mathscr{S}\left(\mathbb{R}^{4 V}\right)$ with supp $f \subset \Omega$. By $\|f\|_{(n)}$ we mean the Schwartz norm of $f:\|f\|_{(n)}=\max _{|\alpha| \leqq n} \max _{x \in \mathbb{R}^{4 V}}\left(1+\underline{x}^{2}\right)^{n / 2}\left|D^{\alpha} f(\underline{x})\right| . \mathscr{S}^{\prime}(\Omega)$ is the dual of $\mathscr{S}(\Omega)$. We define the set:

$$
\Omega_{\neq}^{\mathscr{U}}=\left\{\underline{x} \in \mathbb{R}^{4 V}: x_{v}^{0} \neq x_{v^{\prime}}^{0} \text {, if there is no } \gamma_{i} \in \mathscr{U} \text { with } v, v^{\prime} \in \mathscr{V}\left(\gamma_{i}\right)\right\} \text {. }
$$

Proposition. $\overline{\mathscr{F}}_{(\Gamma, \mathscr{U})}(\underline{x})$ and $\mathscr{E}_{(\Gamma, \mathscr{U})}(\underline{x})$ are elements of $\mathscr{S}^{\prime}\left(\Omega_{\neq}^{\mathscr{U}}\right)$, i.e. there is $M \geqq 0$ such that

$$
\left|\overline{\mathscr{F}}_{(\Gamma, \mathscr{U})}(f)\right| \leqq C\|f\|_{(M)}, \quad f \in \mathscr{P}\left(\Omega_{\neq}^{\mathscr{U}}\right),
$$

and similarly for $\mathscr{E}_{(T, \mathscr{U})}(\underline{x})$.

This proposition is well known, see e.g. $[5,6]$.

\section{Renormalization}

We start with recalling the definition of renormalization in relativistic field theory. The definition below is equivalent to the one given by Hepp [6]:

(Relativistic) renormalization is a mapping $\mathscr{R}$ which assigns to each unrenormalized amplitude $\overline{\mathscr{F}}_{(\Gamma, \mathscr{U})}(\underline{x})$ a tempered distribution $\mathscr{R}_{(\Gamma, \mathscr{\mathscr { F }})}(\underline{x})$ in such a way that certain conditions (conditions R0-R4 below) are satisfied. Let $\tau_{1}, \ldots, \tau_{m} \neq \emptyset$ be subgraphs of $\Gamma$ such that $\tau_{i} \cap \tau_{j}=\emptyset(i \neq j)$, and $\tau_{1} \cup \ldots \cup \tau_{m}=\Gamma$. Define

$$
\left\langle\tau_{1}, \ldots, \tau_{m}\right\rangle_{(\Gamma, \mathscr{U})}^{-}(\underline{x})=\prod_{j=1}^{m} \mathscr{R} \overline{\mathscr{F}}_{\left(\tau_{j}, \mathscr{U}_{\tau_{j}}\right)}\left(\underline{x}_{\tau_{j}}\right) \prod_{\sigma \notin \cup \mathscr{L}\left(\tau_{j}\right)} F_{\sigma}\left(x_{i(\sigma)}-x_{f(\sigma)}\right),
$$

if $\tau_{1}, \ldots, \tau_{m}$ are compatible with $\mathscr{U}$, and

$$
\left\langle\tau_{1}, \ldots, \tau_{m}\right\rangle_{(\Gamma, \mathscr{U})}(\underline{x})=0,
$$


otherwise. Here $F_{\sigma}$ is defined as follows:

$$
F_{\sigma}\left(x_{i(\sigma)}-x_{f(\sigma)}\right)= \begin{cases}\Delta_{+}\left(x_{i(\sigma)}-x_{f(\sigma)}\right), & \text { if } i(\sigma) \in \mathscr{V}\left(\tau_{a}\right), \\ f(\sigma) \in \mathscr{V}\left(\tau_{b}\right), & \text { with } a<b, \\ \Delta_{+}\left(x_{f(\sigma)}-x_{i(\sigma)}\right), & \text { otherwise, }\end{cases}
$$

where $\Delta_{+}(x)=(2 \pi)^{-3} \int d^{4} k \delta\left(k^{2}-\mu^{2}\right) \theta\left(k^{0}\right) e^{-i k x}$. Define also

$$
\mathscr{R} \mathscr{F}_{(\Gamma, \mathscr{U})}(\underline{x})=\sum_{m=1}^{V}(-1)^{V+m} \sum_{\tau_{1}, \ldots, \tau_{m}}\left\langle\tau_{1}, \ldots, \tau_{m}\right\rangle_{(\Gamma, \mathscr{U})}^{-}(\underline{x}) .
$$

Now we formulate the conditions for $\mathscr{R}$.

R0. $\mathscr{R} \overline{\mathscr{F}}_{(\Gamma, \mathscr{U})}(\underline{x})$ is an extension of $\overline{\mathscr{F}}_{(\Gamma, \mathscr{U})}(\underline{x})$ from $\mathscr{S}^{\prime}\left(\Omega_{\neq}^{\mathscr{U}}\right)$ to $\mathscr{S}^{\prime}\left(\mathbb{R}^{4 V}\right) \cdot \mathscr{R}\left[\Lambda_{\Gamma}^{R}(\underline{x})\right]$ $=\Lambda_{\Gamma}^{R}(\underline{x})$.

R1. $\operatorname{supp} \mathscr{R} \overline{\mathscr{F}}_{(\Gamma, \mathscr{U})}(\underline{x}) \subset \Delta^{\mathscr{U}} \equiv\left\{\underline{x} \in \mathbb{R}^{4 V}: x_{v}=x_{v^{\prime}}\right.$, if $v, v^{\prime} \in \mathscr{V}\left(\gamma_{i}\right)$, for some $\left.\gamma_{i} \in \mathscr{U}\right\}$.

$\mathrm{R} 2 . \mathscr{R} \overline{\mathscr{F}}_{(\Gamma, \mathscr{U})}(\underline{x})$ is invariant with respect to the proper Poincare group.

R3. $\mathscr{R}$ is unitary: $\mathscr{R} \overline{\mathscr{F}}_{(\Gamma, \mathscr{U})}(\underline{x})^{*}=\mathscr{R}_{\mathscr{F}}(\Gamma, \mathscr{U})(\underline{x})$.

R4. $\mathscr{R}$ is causal: For any $\emptyset \neq \gamma, \gamma^{\prime} \subset \Gamma$ compatible with $\mathscr{U}$, and such that $\gamma \cap \gamma^{\prime}=\emptyset, \gamma \cup \gamma^{\prime}=\Gamma$, we have

$$
\mathscr{R} \overline{\mathscr{F}}_{(\Gamma, \mathscr{U})}(\underline{x})=\left\langle\gamma, \gamma^{\prime}\right\rangle_{(\Gamma, \mathscr{U})}^{-}(\underline{x}),
$$

if $\underline{x} \in\left\{\underline{x} \in \mathbb{R}^{4 V}: x_{v}-x_{v^{\prime}} \notin \bar{V}_{+}\right.$, if $\left.v \in \mathscr{V}(\gamma), v^{\prime} \in \mathscr{V}\left(\gamma^{\prime}\right)\right\}$.

Later we shall need the following non-covariant version of R4. Denote by

$$
\Omega_{\gamma, \gamma^{\prime}}=\left\{\underline{x} \in \mathbb{R}^{4 V}: x_{v}^{0}<x_{v^{\prime}}^{0} \text {, if } v \in \mathscr{V}(\gamma), v^{\prime} \in \mathscr{V}\left(\gamma^{\prime}\right)\right\} .
$$

R4'. (III.1) holds on $\mathscr{S}\left(\Omega_{\gamma, \gamma^{\prime}}\right)$.

For the sake of convenience we reformulate also the unitary condition.

Let $\iota_{(I, \mathscr{U})}^{(\nu)}(\underline{x})(v \in \mathscr{V})$ be the retarded function (see e.g. [3])

$$
\begin{aligned}
r_{(\Gamma, \mathscr{U})}^{(\nu)}(\underline{x})= & \sum_{\substack{\gamma, \gamma^{\prime} \subset \Gamma \\
v \in \mathscr{V}(\gamma)}}(-1)^{|\mathscr{V}(\gamma)| \mathscr{R}_{\left(\gamma, \mathscr{F}_{\gamma}\right)}}\left(\underline{x}_{\gamma}\right) \mathscr{R} \mathscr{F}_{\left(\gamma^{\prime}, \mathscr{U}_{\gamma^{\prime}}\right)}\left(\underline{x}_{\gamma^{\prime}}\right) \\
& \cdot \prod_{\substack{\sigma: i(\sigma) \in \mathscr{V}(\gamma) \\
f(\sigma) \in \mathscr{V}\left(\gamma^{\prime}\right)}} \Delta_{+}\left(x_{i(\sigma)}-x_{f(\sigma)}\right),
\end{aligned}
$$

where the summation extends over $\mathscr{U}$-compatible $\gamma, \gamma^{\prime} \subset \Gamma$ such that $\gamma \cap \gamma^{\prime}=\emptyset$, $\gamma \cup \gamma^{\prime}=\Gamma$ and $v \in \mathscr{V}(\gamma)$. R3 and the relation

imply that

$$
\begin{aligned}
& \sum_{\gamma, \gamma^{\prime} \subset \Gamma}(-1)^{|\mathscr{V}(\gamma)|} \mathscr{R}_{\left(\gamma, \mathscr{U}_{\gamma}\right)}\left(\underline{x}_{\gamma}\right) \mathscr{R} \mathscr{F}_{\left(\gamma^{\prime}, \mathscr{U}_{\gamma^{\prime}}\right)}\left(\underline{x}_{\gamma^{\prime}}\right) \\
& \quad \cdot \prod_{\substack{\sigma: i(\sigma) \in \mathscr{V}(\gamma) \\
f(\sigma) \in \mathscr{V}\left(\gamma^{\prime}\right)}} \Delta_{+}\left(x_{i(\sigma)}-x_{f(\sigma)}\right)=0
\end{aligned}
$$

$$
r_{(\Gamma, \mathscr{U})}^{(\imath)}(\underline{x})^{*}=(-1)^{V+1_{(\Gamma, \mathscr{U})}^{(\nu)}}(\underline{x}) .
$$

Conversely, (III.2) implies R3. If $\hat{\imath}_{(\Gamma, \mathscr{U})}^{(v)}(p)$ is the Fourier transform $\left[\right.$ with $\delta\left(\sum_{j=1}^{V} p_{j}\right)$ omitted $]$ of $\imath_{(r, \mathscr{U})}^{(v)}(\underline{x})$, then (III.2) reads:

$$
\hat{\imath}_{(\Gamma, \mathscr{U})}^{(\imath)}(p)^{*}=(-1)^{V+1} \hat{\varepsilon}_{(\Gamma, \mathscr{U})}^{(\vartheta)}(-p) .
$$


(Euclidean) renormalization is a mapping which assigns to each unrenormalized Euclidean amplitude $\mathscr{E}_{(\Gamma, \mathscr{U})}(\underline{x})$ a tempered distribution $\mathscr{T}_{\mathscr{E}} \mathscr{E}_{(\Gamma, \mathscr{U})}(\underline{x})$ satisfying the following conditions:

E0. $\mathscr{T}_{\mathscr{E}}(\Gamma, \mathscr{U})(\underline{x})$ is an extension of $\mathscr{E}_{(\Gamma, \mathscr{U})}(\underline{x})$ from $\mathscr{S}^{\prime}\left(\Omega_{\neq}^{\mathscr{U}}\right)$ to $\mathscr{S}^{\prime}\left(\mathbb{R}^{4 V}\right)$. $\mathscr{T}\left[\Lambda_{\Gamma}^{E}(\underline{x})\right]=\Lambda_{\Gamma}^{E}(\underline{x})$.

E1. $\operatorname{supp} \mathscr{T}_{\mathscr{E}}^{\mathscr{E}}(\Gamma, \mathscr{U})(\underline{x}) \subset \Delta^{\mathscr{U}}$.

E2. $\mathscr{T}_{\mathscr{E}}^{\mathscr{E}}(\Gamma, \mathscr{U})(\underline{x})$ is invariant with respect to the inhomogeneous Euclidean group.

E3. $\mathscr{T} \mathscr{E}_{(\Gamma, \mathscr{U})}(\underline{x})^{*}=\mathscr{T} \mathscr{E}_{(\Gamma, \mathscr{U})}(\underline{x})$.

E4. For any $\emptyset \neq \gamma, \gamma^{\prime} \subset \Gamma$ compatible with $\mathscr{U}$ and such that $\gamma \cap \gamma^{\prime}=\emptyset, \gamma \cup \gamma^{\prime}=\Gamma$,

$$
\begin{aligned}
\mathscr{T} \mathscr{E}_{(\Gamma, \mathscr{U})}(\underline{x})= & \mathscr{T} \mathscr{E}_{\left(\gamma, \mathscr{U}_{\gamma}\right)}\left(\underline{x}_{\gamma}\right) \mathscr{T}_{\left.\mathscr{E}_{\left(\gamma^{\prime}\right.} \cdot \mathscr{U}_{\gamma^{\prime}}\right)}\left(\underline{x}_{\gamma^{\prime}}\right) \\
& \cdot \prod_{\substack{\sigma: i(\sigma) \in \mathscr{V}(\gamma) \\
f(\sigma) \in \mathscr{V}\left(\gamma^{\prime}\right)}} S\left(x_{i(\sigma)}-x_{f(\sigma)}\right),
\end{aligned}
$$

if $\underline{x} \in\left\{\underline{x} \in \mathbb{R}^{4 V}: x_{v} \neq x_{v^{\prime}}\right.$, if $\left.v \in \mathscr{V}(\gamma), v^{\prime} \in \mathscr{V}\left(\gamma^{\prime}\right)\right\}$.

Again, we formulate an alternative condition:

E4'. (III.4) holds on $\mathscr{S}\left(\Omega_{\gamma, \gamma^{\prime}}\right)$.

Remark that E3 and R3 though very similar in appearance, are very different in structure. While E3 is a simple reality condition, R3 is a nonlinear condition involving products of Feynman amplitudes.

Lemma. (a) $\left(\mathrm{E} 0, \ldots, \mathrm{E} 4^{\prime}\right) \Leftrightarrow(\mathrm{E} 0, \ldots, \mathrm{E} 4)$,

(b) $\left(\mathrm{R} 0, \ldots, \mathrm{R} 4^{\prime}\right) \Leftrightarrow(\mathrm{R} 0, \ldots, \mathrm{R} 4)$.

The proof of this lemma is given in Appendix A.

\section{The Equivalence Theorem}

Theorem ER. The axioms (R) and (E) are equivalent in the following sense. Given any relativistic (respectively Euclidean) renormalization $\mathscr{R}$ (respectively $\mathscr{T}$ ) there exists precisely one Euclidean (respectively relativistic) renormalization $\mathscr{T}$ (respectively $\mathscr{R}$ ) such that the following condition is satified: For any generalized graph $(\Gamma, \mathscr{U})$ and a radially analytic function $f \in \mathscr{S}\left(\mathbb{R}^{4 V}\right)$ we have

$$
i^{V} \mathscr{T} \mathscr{E}_{(\Gamma, \mathscr{U})}\left(f_{i}\right)=\mathscr{R} \overline{\mathscr{F}}_{(\Gamma, \mathscr{U})}(f) .
$$

For the definition of radially analytic functions see [2] or Appendix B. The strategy of the proof is similar to that of the proof of the basic result of [2]. We use induction with respect to $V$. For $V=1$ there is nothing to prove. Our induction hypothesis is that the equivalence has already been established for all graphs with $V \leqq n-1$. Suppose we are given a relativistic (respectively Euclidean) renormalization. To define the corresponding Euclidean (respectively relativistic) renormalization we decompose a test function $f$ into two parts: the (essentially) radially analytic part $f_{1}$, and the part $f_{0}$ vanishing on the diagonal. Now, to define

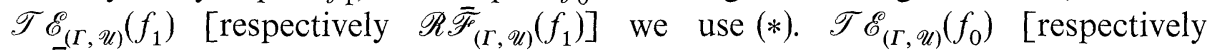
$\left.\mathscr{R} \overline{\mathscr{F}}_{(\Gamma, \mathscr{U})}\left(f_{0}\right)\right]$ is defined with the help of the induction hypothesis. Observe that the uniqueness follows immediately from $(*)$ and Lemma B.1. 


\section{Proof of the Equivalence Theorem: $R \Rightarrow E$}

1. The Decomposition of $f$

Let $K \geqq 0$ be an integer, which will be specified later. Consider the $C^{K}$-function

$$
\alpha_{\lambda, K}(t)=\theta(t) \lambda^{K+1} t^{K+1} e^{(i-1) \lambda t}=\frac{(K+1) !}{2 \pi} \int d p e^{-i p t}\left(1-i-i \lambda^{-1} p\right)^{-K-2},
$$

where $t \in \mathbb{R}, \lambda \in A$ (see Appendix $\mathrm{B}$ for the definition of $\mathrm{A}$ ). It satisfies the equation:

Define

$$
\lambda\left(1-i+\lambda^{-1} \frac{\partial}{\partial t}\right)^{K+2} \alpha_{\lambda, K}(t)=(K+1) ! \delta(t) .
$$

$$
\begin{aligned}
\psi\left(x_{1}^{0}, \underline{\xi}, \underline{\mathbf{x}}\right)= & i[(K+1) !]^{-1}\left(1-i-i \frac{\partial}{\partial x_{1}^{0}}\right)^{K+2} \\
& \cdot f\left(x_{1}^{0}, \mathbf{x}_{1} ; x_{1}^{0}+\xi_{1}, \mathbf{x}_{2} ; \ldots, x_{1}^{0}+\xi_{V-1}, \mathbf{x}_{V}\right),
\end{aligned}
$$

where $\xi_{j}=x_{j+1}^{0}-x_{1}^{0}, j=1, \ldots, V-1$. Then

$$
f(\underline{x})=\int d t \alpha_{i, K}\left(x_{1}^{0}-t\right) \psi(t, \underline{\xi}, \underline{\mathbf{x}}) .
$$

Observe that $\|\psi\|_{(n)} \leqq C\|f\|_{(n+K+2)}$. Let $w(\xi) \in \mathscr{S}\left(\mathbb{R}^{V-1}\right)$ be a radially analytic function such that $w(\underline{0})=1, D^{\alpha} w(\underline{0})=0$, for $0<|\alpha| \leqq K$. [Such a function exists. Proof. By the density of radially analytic functions there is a radially analytic $v(\xi)$ such that $v(\underline{0})=1$. Assume that $D^{\alpha} v(\underline{0})=0$, for all $0<|\alpha| \leqq p-1$. Define

$$
v^{\prime}(\underline{\xi})=v(\underline{\xi})\left[1-\sum_{|\alpha|=p}\left(a_{\alpha} / \alpha !\right) \underline{\xi}^{\alpha}\right],
$$

where $a_{\alpha}=D^{\alpha} v(\underline{0})$. Then $v^{\prime}(\underline{\xi})$ is radially analytic, and $v^{\prime}(\underline{0})=1, D^{\alpha} v^{\prime}(\underline{0})=0$, for all $0<|\alpha| \leqq p$.] Define

$$
f_{1}(\underline{x})=\int d t \alpha_{i, K}\left(x_{1}^{0}-t\right) w(\underline{\xi}) \sum_{|\beta|=0}^{K} \frac{\underline{\xi}^{\beta}}{\bar{\beta} !} D_{\xi}^{\beta} \psi(t, \underline{0}, \underline{\mathbf{x}}),
$$

and $f_{0}(\underline{x})=f(\underline{x})-f_{1}(\underline{x})$. Denote by $\mathscr{S}_{K}(\Omega)(\Omega$ - open) the closure of $\mathscr{S}(\Omega)$ in the norm $\|\cdot\|_{(K)}$. Then $f_{0} \in \mathscr{S}_{K}\left(\Omega_{0}\right), f_{1} \in \mathscr{S}_{K}\left(\mathbb{R}^{4 V}\right)$, where $\Omega_{0}=\left\{\underline{x} \in \mathbb{R}^{4 V}:\right.$ there is a pair $i, j$ $(i \neq j)$ such that $\left.x_{1}^{0} \neq x_{j}^{0}\right\}$. Moreover

$$
\left\|f_{0}\right\|_{(n)} \leqq C\|\psi\|_{(n+K)} \leqq C\|f\|_{(n+2 K+2)}
$$

(a similar bound holds for $f_{1}$ ).

2. The Definition of $\mathscr{T}_{(\Gamma, \mathscr{E})}\left(f_{1}\right)$

Rewrite $f_{1}$ in the form

where

$$
f_{1}(\underline{x})=\int d t \varphi_{1}^{t}\left(x_{1}^{0}-t, \underline{\xi}, \underline{\mathbf{x}}\right)
$$

$$
\begin{aligned}
\varphi_{\lambda}^{t}\left(x_{1}^{0}, \underline{\xi}, \underline{\mathbf{x}}\right)= & \left\{\sum_{|\beta|=0}^{K} \frac{\lambda^{|\beta|} \underline{\underline{\xi}} \beta}{\beta !} D_{\xi}^{\beta} \psi(t, \underline{0}, \underline{\mathbf{x}})\right\} \\
& \cdot w \underline{(\lambda \underline{\xi})} \alpha_{i, K}\left(\lambda x_{1}^{0}\right) ; \quad \lambda \in A^{*} .
\end{aligned}
$$




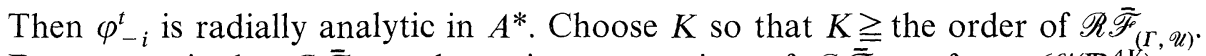
Denote again by $\mathscr{R} \overline{\mathscr{F}}_{(\Gamma, \mathscr{U})}$ the unique extension of $\mathscr{R} \overline{\mathscr{F}}_{(\Gamma, \mathscr{U})}$ from $\mathscr{S}^{\prime}\left(\mathbb{R}^{4 V}\right)$ to $\mathscr{S}_{K}^{\prime}\left(\mathbb{R}^{4 V}\right)$. Define

$$
\mathscr{T} \mathscr{E}_{(\Gamma, \mathscr{U})}\left(\varphi_{1}^{t}\right)=i^{-V} \mathscr{R} \tilde{\mathscr{F}}_{(\Gamma, \mathscr{U})}\left(\varphi_{-i}^{t}\right)
$$

We have

$$
\left|\mathscr{R} \overline{\mathscr{F}}_{(\Gamma, \mathscr{U})}\left(\varphi_{-i}^{t}\right)\right| \leqq C\left\|\varphi_{-i}^{t}\right\|_{(K)} \leqq C\left(1+t^{2}\right)^{-1}\|f\|_{(3 K+2)} .
$$

Hence, we are allowed to integrate (V.2) over $t$. Set

$$
\mathscr{T} \mathscr{E}_{(\Gamma, \mathscr{U})}\left(f_{1}\right)=i^{-V} \int d t \mathscr{R} \overline{\mathscr{F}}_{(\Gamma, \mathscr{U})}\left(\varphi_{-i}^{t}\right) \text {. }
$$

Clearly $\left|\mathscr{T}_{\mathscr{E}_{(\Gamma, \mathscr{U})}}\left(f_{1}\right)\right| \leqq C\|f\|_{(3 K+2)}$.

\section{The Definition of $\mathscr{T}_{(} \mathscr{E}_{(\Gamma, \mathscr{U})}\left(f_{0}\right)$}

If $\mathscr{U}=\{\Gamma\}$, then we set $\mathscr{T}_{\mathscr{E}}^{\mathscr{E}}(\Gamma, \mathscr{U})\left(f_{0}\right)=0$. Otherwise, for any pair of subgraphs $\emptyset \neq \gamma$, $\gamma^{\prime} \subset \Gamma$, such that $\gamma \cap \gamma^{\prime}=\emptyset, \gamma \cup \gamma^{\prime}=\Gamma$, we define a distribution $\mathscr{G}_{\left(\gamma, \gamma^{\prime}\right)} \in \mathscr{S}^{\prime}\left(\Omega_{\left(\gamma, \gamma^{\prime}\right)}\right)$ as

$$
\left\{\begin{array}{l}
\text { the right-hand side of (III.4), if } \gamma, \gamma^{\prime} \text { are compatible with } \mathscr{U}, \\
0, \text { otherwise. }
\end{array}\right.
$$

It follows from the induction hypothesis that $\mathscr{G}_{\left(\gamma, \gamma^{\prime}\right)}$ has the following property:

$$
\mathscr{G}_{\left(\gamma, \gamma^{\prime}\right)}(\underline{x})=\mathscr{G}_{\left(\tau, \tau^{\prime}\right)}(\underline{x})
$$

if $\underline{x} \in \Omega_{\gamma, \gamma^{\prime}} \cap \Omega_{\tau, \tau^{\prime}}$

Lemma. Every $f \in \mathscr{S}\left(\Omega_{0}\right)$ can be decomposed as follows

$$
f=\sum_{\gamma, \gamma^{\prime}} f_{\left(\gamma, \gamma^{\prime}\right)}
$$

where supp $f_{\left(\gamma, \gamma^{\prime}\right)} \subset \Omega_{\gamma, \gamma^{\prime}}$, and $\gamma, \gamma^{\prime}$ run through all the pairs of subgraphs of $\Gamma$ such that $\gamma \cap \gamma^{\prime}=\emptyset, \gamma \cup \gamma^{\prime}=\Gamma ; \gamma, \gamma^{\prime} \neq \emptyset$. Moreover

(i) $\left\|f_{\left(\gamma, \gamma^{\prime}\right)}\right\|_{(n)} \leqq C\|f\|_{(n)}$,

(ii) if supp $f \subset \Omega$, then $\operatorname{supp} f_{\left(\gamma, \gamma^{\prime}\right)} \subset \Omega \cap \Omega_{\gamma, \gamma^{\prime}}$,

(iii) if $f$ is radially analytic, then so is $f_{\left(\gamma, \gamma^{\prime}\right)}$.

The proof of this lemma is standard (cf. [2, p. 105]). Define

$$
\mathscr{G}_{(\Gamma, \mathscr{U})}(f)=\sum_{\gamma, \gamma^{\prime}} \mathscr{G}_{\left(\gamma, \gamma^{\prime}\right)}\left(f_{\left(\gamma, \gamma^{\prime}\right)}\right) .
$$

By (i) of the lemma $\mathscr{G}_{(\Gamma, \mathscr{U})}\left(\underline{\underline{x})}\right.$ is a distribution in $\mathscr{S}^{\prime}\left(\Omega_{0}\right)$ of order (say) $N$. By (V.3), and (ii) of the lemma it satisfies

$$
\mathscr{G}_{(\Gamma, \mathscr{U})}(f)=\mathscr{G}_{\left(\gamma, \gamma^{\prime}\right)}(f),
$$

if supp $f \subset \Omega_{\gamma, \gamma^{\prime}}$. It follows from the definition that $\mathscr{G}_{(\Gamma, \mathscr{U})}$ is the only distribution in $\mathscr{S}^{\prime}\left(\Omega_{0}\right)$ satisfying (V.5). Denoting again by $\mathscr{G}_{(\Gamma, \mathscr{U})}$ the extension of $\mathscr{G}_{(\Gamma, \mathscr{U})}$ to $\mathscr{S}_{K}^{\prime}\left(\Omega_{0}\right)$ (we choose $K \geqq N$ ), we define $\mathscr{T}_{\mathscr{E}}^{\mathscr{E}_{(T, \mathscr{U})}}\left(f_{0}\right)=\mathscr{G}_{(\Gamma, \mathscr{U})}\left(f_{0}\right)$. By $(\mathrm{V} .1)$

$$
\left|\mathscr{T} \mathscr{E}_{(\Gamma, \mathscr{U})}\left(f_{0}\right)\right| \leqq C\|f\|_{(3 K+2)} .
$$




\section{Verification of the Axioms}

We set

$$
\mathscr{T} \mathscr{E}_{(\Gamma, \mathscr{U})}(f)=\mathscr{T} \mathscr{E}_{(\Gamma, \mathscr{U})}\left(f_{0}\right)+\mathscr{T} \mathscr{E}_{(\Gamma, \mathscr{U})}\left(f_{1}\right) .
$$

It follows from the estimates of 2. and 3. that $\left|\mathscr{T} \mathscr{E}_{(\Gamma, \mathscr{U})}(f)\right| \leqq C\|f\|_{(3 K+2)}$, i.e. $\mathscr{T}_{\mathscr{E}}^{\mathscr{E}_{(\Gamma, \mathscr{U})}} \in \mathscr{S}^{\prime}\left(\mathbb{R}^{4 V}\right)$. If $f \in \mathscr{S}\left(\Omega_{\neq}^{\mathscr{U}}\right)$, then $f_{1}=0$, and hence $\mathscr{T}_{\mathscr{E}}^{\mathscr{E}}(\Gamma, \mathscr{U})(\underline{x})$ is an extension of $\mathscr{E}_{(\Gamma, \mathscr{U})}(\underline{x})$. This proves E0. E4 $4^{\prime}$ is clear: If $\operatorname{supp} f \subset \Omega_{\gamma, \gamma^{\prime}}\left(\gamma, \gamma^{\prime}\right.$ compatible with $\left.\mathscr{U}\right)$, then $f_{1}=0$, and

$$
\mathscr{T}_{(\Gamma, \mathscr{U})}(f)=\mathscr{G}_{(\Gamma, \mathscr{U})}(f)=\mathscr{G}_{\left(\gamma, \gamma^{\prime}\right)}(f) .
$$

We prove $(*)$. Let $f$ be radially analytic. Then also $f_{0}$ and $f_{1}$ are radially analytic. For $f=f_{1}(*)$ follows directly from the construction. If $f=f_{0}$, then by the lemma it suffices to consider $f=f_{0,\left(\gamma, \gamma^{\prime}\right)}$ only. We have

$$
\begin{aligned}
& i^{V} \int \mathscr{T} \mathscr{E}_{(\Gamma, \mathscr{U})}(\underline{x}) f_{i}(\underline{x}) d \underline{x} \\
& =i^{V} \int \mathscr{T} \mathscr{E}_{\left(\gamma, \mathscr{U}_{\gamma}\right)}\left(\underline{x}_{\gamma}\right) \mathscr{T} \mathscr{E}_{\left(\gamma^{\prime}, \mathscr{U}_{\gamma^{\prime}}\right)}\left(\underline{x}_{\gamma^{\prime}}\right) \prod_{\substack{\sigma: i(\sigma) \in \mathscr{V}(\gamma) \\
f(\sigma) \in \mathscr{V}\left(\gamma^{\prime}\right)}} S\left(x_{i(\sigma)}-x_{f(\sigma)}\right) f_{i}(\underline{x}) d \underline{x} \\
& =\int \mathscr{R} \overline{\mathscr{F}}_{\left(\gamma, \mathscr{U}_{\gamma}\right)}\left(\underline{x}_{\gamma}\right) \mathscr{R} \overline{\mathscr{F}}_{\left(\gamma^{\prime}, \mathscr{U}_{\gamma^{\prime}}\right)}\left(\underline{x}_{\gamma^{\prime}}\right) \prod_{\substack{\sigma: i(\sigma) \in \mathscr{V}(\gamma) \\
f(\sigma) \in \mathscr{V}\left(\gamma^{\prime}\right)}} \Delta_{+}\left(x_{i(\sigma)}-x_{f(\sigma)}\right) f(\underline{x}) d \underline{x} \\
& =\int \mathscr{R} \overline{\mathscr{F}}_{(\Gamma, \mathscr{U})}(\underline{x}) f(\underline{x}) d \underline{x},
\end{aligned}
$$

where we have used the induction hypothesis, E4', R4', and the fact that $\left(\Delta_{+}\right)_{i}(x)$ $=S(x)$ for $x^{0}<0$. To prove E2 let us define the following differential operators:

$$
\begin{aligned}
& \left(M^{0 \mu} f\right)(\underline{x})=\sum_{j=1}^{V}\left(x_{j}^{\mu} \frac{\partial}{\partial x_{j}^{0}}-x_{j}^{0} \frac{\partial}{\partial x_{j}^{\mu}}\right) f(\underline{x}), \\
& \left(m^{0 \mu} f\right)(\underline{x})=\sum_{j=1}^{V}\left(x_{j}^{\mu} \frac{\partial}{\partial x_{j}^{0}}+x_{j}^{0} \frac{\partial}{\partial x_{j}^{\mu}}\right) f(\underline{x}),
\end{aligned}
$$

where $\mu=1,2,3$. Then, by Lemma B2 $\left(M^{0 \mu} f\right)_{i}(\underline{x})=i\left(m^{0 \mu} f_{i}\right)(\underline{x})$. Hence, by $(*)$ (just proved)

$$
0=\mathscr{R} \overline{\mathscr{F}}_{(\Gamma, \mathscr{U})}\left(M^{0 \mu} f\right)=i^{V+1} \mathscr{T}_{\mathscr{E}}(\Gamma, \mathscr{U})\left(m^{0 \mu} f_{i}\right)
$$

i.e. $\mathscr{T} \mathscr{E}_{(\Gamma, \mathscr{U})}\left(m^{0 \mu} f\right)=0$, for all $f$ 's radially analytic in $A^{*}$. Since such functions are dense in $\mathscr{S}\left(\mathbb{R}^{4 V}\right)$, the last equality holds for any $f \in \mathscr{S}\left(\mathbb{R}^{4 V}\right)$. From the construction it is clear that $\mathscr{T} \mathscr{E}_{(T, \mathscr{U})}(\underline{x})$ is translation invariant. This proves E2. E1: Take a function $f$ with supp $f \subset\left\{\underline{x} \in \mathbb{R}^{4 V}: x_{v}^{0}=x_{v^{\prime}}^{0} \text {, if } v, v^{\prime} \in \mathscr{V}\left(\gamma_{i}\right) \text { for some } \gamma_{i} \in \mathscr{U}\right\}^{c}$. Then

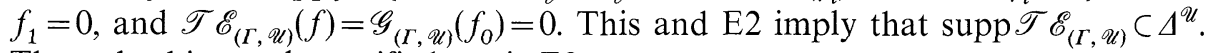
The only thing to be verified yet is E3.

\section{The p-Space Analytic Function}

The set of distributions $\left\{\left\langle\tau_{1}, \ldots, \tau_{m}\right\rangle_{(\Gamma, \mathscr{U})}^{-}(\underline{x})\right\}$ is a perturbative (graph by graph) counterpart of a linear system of $V$-point functions [4]. In fact, it is easy to see that the (perturbative analogue of the) set of axioms of [4], Sect. 6.1, is satisfied. We 
denote the corresponding generalized retarded functions by $i_{(\Gamma, \mathscr{U})}^{\mathscr{S}}(\underline{x})$, where $\mathscr{S}$ runs through the set of all cells of $\mathbb{R}^{V}[4]$. Let $\hat{\imath}_{(\Gamma, \mathscr{C})}^{\mathscr{S}}(\underline{p})$ be the Fourier transform $\left[\right.$ with $\delta\left(\sum_{j=1}^{V} p_{j}\right)$ omitted $]$ of $r_{(\Gamma, \mathscr{U})}^{\mathscr{S}}(\underline{x})$. It is well known that for any $\mathscr{S}_{1}, \mathscr{S}_{2}$ one has

$$
i_{(\Gamma, \mathscr{U})}^{\mathscr{S}_{1}}(\underline{p})=r_{(\Gamma, \mathscr{U})}^{\mathscr{S}_{2}}(\underline{p})
$$

in the open region of $\mathbb{R}^{4(V-1)}$

$$
\bigcap_{J \subset\{1, \ldots, V\}}\left\{\underline{p} \in \mathbb{R}^{4 V}: \sum_{j=1}^{V} p_{j}=0, p_{J}^{2}<\mu^{2}\right\},
$$

where $p_{J}=\sum_{j \in J} p_{j} \cdot r_{(\Gamma, \mathscr{U})}^{\mathscr{S}}(\underline{x})$ has support in a certain cone [4]. Hence, $\hat{r}_{(\Gamma, \mathscr{U})}^{\mathscr{S}}(\underline{p})$ is a boundary value of a function holomorphic in the corresponding tube. From (V.6) and the edge of the wedge theorem it follows that the $r^{\mathscr{S}}$ 's are boundary values (from the corresponding tubes) of a single holomorphic function denoted by $H_{(\Gamma, \mathscr{U})}(\underline{z})$. The domain of holomorphy of $H_{(\Gamma, \mathscr{U})}(\underline{z})$ contains a complex neighbourhood of (V.7).

\section{Verification of $\mathrm{E} 3: \mu^{2}>0$}

In this case the domain of holomorphy of $H_{(\Gamma, \mathscr{U})}(\underline{z})$ contains the Euclidean momenta $\underline{z}=\left(\left(-i p_{1}^{0}, \mathbf{p}_{1}\right), \ldots,\left(-i p_{V}^{0}, \mathbf{p}_{V}\right)\right)$. Using a suitable regularization one shows as in $[2,4]$, that

$$
i^{V-1} \int d \underline{p} e^{-i \sum p_{j} x_{j}} H_{(\Gamma, \mathscr{U})}\left(\left(-i p_{1}^{0}, \mathbf{p}_{1}\right), \ldots,\left(-i p_{V}^{0}, \mathbf{p}_{\Gamma}\right)\right) \delta\left(\sum_{j=1}^{V} p_{j}\right)
$$

satisfies $(*)$, i.e.

$$
\mathscr{T} \mathscr{E}_{(\Gamma, \mathscr{U})}(\underline{x})=i^{V-1} \int d \underline{p} e^{-i \Sigma p_{j} x_{j}} H_{(\Gamma, \mathscr{U})}\left(\left(-i p_{1}^{0}, \mathbf{p}_{1}\right), \ldots,\left(-i p_{V}^{0}, \mathbf{p}_{V}\right)\right) \delta\left(\sum_{j=1}^{V} p_{j}\right) .
$$

We shall show that

$$
\begin{aligned}
& H_{(\Gamma, \mathscr{U})}\left(\left(-i p_{1}^{0}, \mathbf{p}_{1}\right), \ldots,\left(-i p_{V}^{0}, \mathbf{p}_{V}\right)\right) \\
& \quad=(-1)^{V+1} H_{(\Gamma, \mathscr{U})}\left(\left(i p_{1}^{0},-\mathbf{p}_{1}\right), \ldots,\left(i p_{V}^{0},-\mathbf{p}_{V}\right)\right),
\end{aligned}
$$

which is equivalent to E3. The points $\left(\left(0, \mathbf{p}_{1}\right), \ldots,\left(0, \mathbf{p}_{V}\right)\right)$ are in $(\mathrm{V} .7)$, and

$$
\begin{aligned}
& \left.D^{\alpha} H_{(\Gamma, \mathscr{U})}\left(\left(-i p_{1}^{0}, \mathbf{p}_{1}\right), \ldots,\left(-i p_{V}^{0}, \mathbf{p}_{V}\right)\right)\right|_{\underline{p}^{0}=0} \\
& \quad=\left.(-i)^{\left|\alpha_{0}\right|} D^{\alpha} r_{(\Gamma, \mathscr{U})}^{\mathscr{S}}(\underline{p})\right|_{\underline{p}^{0}=\underline{0}},
\end{aligned}
$$

for any $\alpha$. Here $\left|\alpha_{0}\right|=\sum \alpha_{j}^{0}$. The function $F(p) \equiv H_{(\Gamma, \mathscr{U})}\left(\left(-i p_{1}^{0}, \mathbf{p}_{1}\right), \ldots,\left(-i p_{V}^{0}, \mathbf{p}_{V}\right)\right)^{*}$ $-(-1)^{V+1} H_{(\Gamma, \mathscr{U})}\left(\left(i p_{1}^{0},-\mathbf{p}_{1}\right), \ldots,\left(i p_{V}^{0},-\mathbf{p}_{V}\right)\right)$ is real analytic. By (V.9), (III.3), and (V.6) $D^{\alpha} F(\underline{0})=0$. Thus $F(\underline{p}) \equiv 0$ in a neighbourhood of $\underline{0}$, and hence $F(\underline{p}) \equiv 0$ for all $\underline{p} \in \mathbb{R}^{4 V}$. 


\section{Verification of $\mathrm{E} 3: \mu^{2} \geqq 0$}

As in [2] we consider the function:

$$
F_{A}(z)=\exp \left\{i A^{-1}\left[z^{2}-A^{2}\right]^{1 / 2}+1\right\}, \quad A>0,
$$

where $z \in \mathbb{C}^{4}, z^{2} \notin\left[A^{2}, \infty\right.$ ) (we choose that branch of the square root, which has a positive imaginary part in $\mathbb{C} \backslash\left[A^{2}, \infty\right)$ ). Denote

$$
\begin{gathered}
F_{A}^{+}(x)=\lim _{V+\ni y \rightarrow 0} F_{A}(x-i y), \\
\bar{F}_{A}(x)=\theta\left(-x^{0}\right) F_{A}^{+}(x)+\theta\left(x^{0}\right) F_{A}^{+}(-x) .
\end{gathered}
$$

Similarly as in [4, Sect. 6.3] we define a new (regularized) linear system:

$$
\left\langle\tau_{1}, \ldots, \tau_{m}\right\rangle_{(\Gamma, \mathscr{U})}^{A}(\underline{x})=\prod_{\sigma \in \mathscr{L} \backslash \cup \mathscr{L}\left(\gamma_{i}\right)} F_{A}^{(?)}\left(x_{i(\sigma)}-x_{f(\sigma)}\right)\left\langle\tau_{1}, \ldots, \tau_{m}\right\rangle_{(\Gamma, \mathscr{U})}^{-}(\underline{x}),
$$

where

$$
\begin{aligned}
F_{A}^{(?)}\left(x_{i(\sigma)}-x_{f(\sigma)}\right)= & \bar{F}_{A}\left(x_{i(\sigma)}-x_{f(\sigma)}\right), \quad \text { if } \quad \sigma \in \mathscr{L}\left(\tau_{j}\right), \text { for some } j, \\
& F_{A}^{+}\left(x_{i(\sigma)}-x_{f(\sigma)}\right), \quad \text { if } \quad i(\sigma) \in \mathscr{V}\left(\tau_{j}\right), \\
& f(\sigma) \in \mathscr{V}\left(\tau_{k}\right), \quad \text { with } j<k, \\
& F_{A}^{+}\left(x_{f(\sigma)}-x_{i(\sigma)}\right), \quad \text { if } \quad i(\sigma) \in \mathscr{V}\left(\tau_{j}\right), \\
& f(\sigma) \in \mathscr{V}\left(\tau_{k}\right), \quad \text { with } j>k .
\end{aligned}
$$

Then $\left\langle\tau_{1}, \ldots, \tau_{m}\right\rangle_{(\Gamma, \mathscr{U})}^{A}(\underline{x})$ have positive thresholds $\geqq A^{-1}$, and $\left\langle\tau_{1}, \ldots, \tau_{m}\right\rangle_{(\Gamma, \mathscr{U})}^{A}$ $\rightarrow\left\langle\tau_{1}, \ldots, \tau_{m}\right\rangle_{(\Gamma, \mathscr{U})}^{-}$as $A \rightarrow \infty$. As in 2. and 3. we construct the corresponding $\mathscr{T} \mathscr{E}_{(\Gamma, \mathscr{U})}(\underline{x})$. It follows from the construction that $\mathscr{T}_{(\Gamma, \mathscr{U})} \rightarrow \mathscr{T}_{\mathscr{E}} \mathscr{E}_{(\Gamma, \mathscr{U})}$. Repeating the argument of 5. and 6., we prove that $\mathscr{T}_{\mathscr{E}}^{\left.\mathscr{E}_{(}^{A}, \mathscr{U}\right)}(\underline{x})^{*}=\mathscr{T}_{\mathscr{E} A} \mathscr{E}_{(\Gamma, \mathcal{U})}(\underline{x})$. This implies E3.

\section{Proof of the Equivalence Theorem: $E \Rightarrow R$}

We sketch only the argument, because it is based on the same principle as the proof of $R \Rightarrow E$.

1. Any function $f \in \mathscr{S}\left(\mathbb{R}^{4 V}\right)$ can be decomposed as $f_{0}+f_{1}$, where

$$
f_{1}(\underline{x})=\int d t \alpha_{1, K}\left(x_{1}^{0}-t\right) w_{i}(\underline{\xi}) \sum_{|\beta|=0}^{K} \frac{\underline{\xi}^{\beta}}{\overline{\beta !}} D_{\xi}^{\beta} \psi(t, \underline{0}, \underline{\mathbf{x}}) .
$$

Here

$$
\begin{aligned}
\psi\left(x_{1}^{0}, \underline{\xi}, \underline{\mathbf{x}}\right)= & {[(K+1) !]^{-1}\left(1-i-\frac{\partial}{\partial x_{1}^{0}}\right)^{K+2} } \\
& \cdot f\left(x_{1}^{0}, \mathbf{x}_{1} ; x_{1}^{0}+\xi_{1}, \mathbf{x}_{2} ; \ldots ; x_{1}^{0}+\xi_{V-1}, \mathbf{x}_{V}\right) .
\end{aligned}
$$

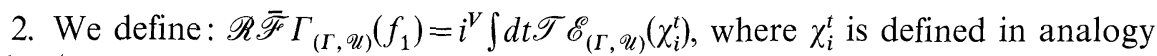
with $\varphi_{\lambda}^{t}$.

3. Using the induction hypothesis and proceeding as in the proof of $R \Rightarrow E$ we construct the relativistic counterpart of $\mathscr{G}_{(\Gamma, \mathscr{U})}$, denoted by $\overline{\mathscr{G}}_{(\Gamma, \mathscr{U})}$. Define: $\mathscr{R} \overline{\mathscr{F}}_{(\Gamma, \mathscr{U})}\left(f_{0}\right)=\overline{\mathscr{G}}_{(\Gamma, \mathscr{U})}\left(f_{0}\right)$. 
4. We verify the axioms (without unitarity) and (*).

5., 6. We verify unitarity for $\mu^{2}>0$. Using the already proved properties of $\mathscr{R} \overline{\mathscr{F}}_{(\Gamma, \mathscr{U})}(\underline{x})$ we repeat the construction of 5., 6., Sect. V. Then E3 implies (V.8). Using (V.9) we prove (III.3) in some ball around $\underline{0}$ contained in (V.7). Hence $H_{(\Gamma, \mathscr{U})}(\underline{z})^{*}=(-1)^{V+1} H_{(\Gamma, \mathscr{U})}\left(-\underline{z}^{*}\right)$ in the domain of holomorphy. Passing to boundary values we obtain $(*)$.

7. We proceed precisely as in 7., Sect. V. Q.E.D.

\section{Existence of Renormalization}

Theorem E. There exists a Euclidean renormalization. It is unique up to a finite renormalization.

Proof. We use induction with respect to $V$. Our induction hypothesis is that $\mathscr{T}$ has already been constructed for all $(\Gamma, \mathscr{V})$ with $V \leqq n-1$. Let $V=n$. If $\mathscr{V}=\{\Gamma\}$, then we define $\mathscr{T} \mathscr{E}_{(\Gamma, \mathscr{U})}(\underline{x})=\mathscr{E}_{(\Gamma, \mathscr{U})}(\underline{x})=\Lambda_{\Gamma}^{E}(\underline{x})$. Suppose that $\mathscr{V}$ contains at least two elements. Define the sets: $\Xi_{\gamma, \gamma^{\prime}}=\left\{\underline{x} \in \mathbb{R}^{4 V}: x_{v} \neq x_{v^{\prime}}\right.$, if $\left.v \in \mathscr{V}(\gamma), v^{\prime} \in \mathscr{V}(\gamma)\right\}$, and $\Xi_{0}=\left\{\underline{x} \in \mathbb{R}^{4 V}\right.$ : there is a pair $i, j(i \neq j)$ such that $\left.x_{i} \neq x_{j}\right\}$. Similarly as in 3., Sect. V we construct a unique distribution $\mathscr{H}_{(\Gamma, \mathscr{U})} \in \mathscr{S}^{\prime}\left(\Xi_{0}\right)$, such that

$$
\mathscr{H}_{(\Gamma, \mathscr{U})}(\underline{x})=\mathscr{H}_{\left(\gamma, \gamma^{\prime}\right)}(\underline{x}), \quad \text { if } \quad x \in \Xi_{\gamma, \gamma^{\prime}} .
$$

Here $\mathscr{H}_{\left(\gamma, \gamma^{\prime}\right)}(\underline{x})$ is defined as the right hand side of (III.4), if $\gamma, \gamma^{\prime}$ are compatible with $\mathscr{U}$, and $\mathscr{H}_{(\gamma, \gamma)}(\underline{x})=0$, otherwise. Clearly $\mathscr{H}_{(\Gamma, \mathscr{U})}(\underline{x})$ is Euclidean invariant.

Let $\varphi \in \mathscr{S}\left(\mathbb{R}^{4(v-1)}\right)$ be a fixed real valued function such that (i) $\varphi(\underline{0})=1$, (ii) $D^{\alpha} \varphi(\underline{0})=0$, for all $|\alpha| \leqq N\left(N=\right.$ the order of $\left.\mathscr{H}_{(\Gamma, \mathscr{U})}\right)$. Denote by $\zeta_{j}=x_{j+1}-x_{1}$, $j=1, \ldots, V-1$, and

$$
(R f)(\underline{x})=f(\underline{x})-\varphi(\underline{\zeta}) \sum_{|\alpha| \leqq N} \frac{\zeta^{\alpha}}{\alpha !} D_{\zeta}^{\alpha} f\left(x_{1}, \ldots, x_{1}\right),
$$

for $f \in \mathscr{S}\left(\mathbb{R}^{4 V}\right)$. Then $R f \in \mathscr{S}_{N}\left(\Xi_{0}\right)$, and

$$
\|R f\|_{(n)} \leqq C\|f\|_{(n+N)} .
$$

Let $O(4)$ denote the homogeneous Euclidean group, $\mu$ the normalized Haar measure on $O(4)$. For $g \in O(4)$ we denote $\left(g \mathscr{H}_{(\Gamma, \mathscr{U})}\right)(f)=\mathscr{H}_{(\Gamma, \mathscr{U})}(g f)$, where $(g f)(\underline{x})$ $=f\left(\underline{\left.g^{-1} x\right)}\right.$. Define

$$
\mathscr{T}_{(\Gamma, \mathscr{U})}(f)=\int_{O(4)} d \mu(g)\left(g \mathscr{H}_{(\Gamma, \mathscr{U})}\right)(R f)
$$

We verify that the so defined $\mathscr{T} \mathscr{E}_{(\Gamma, \mathscr{U})}$ satisfies conditions E0-E4. E0 follows from (VII.2) and the fact that $R f=f$, if $f \in \mathscr{S}\left(\Omega_{\neq}^{\mathscr{U}}\right) . \mathscr{T}_{\mathscr{E}}^{\mathscr{E}}(\Gamma, \mathscr{U})$ is rotation invariant because of the invariance of the Haar measure. It is clear from the definition that $\mathscr{T} \mathscr{E}_{(T, \mathscr{U})}$ is translation invariant. This establishes E2. E1 and E4 follow from (VII.1) and the fact that $g\left(\Xi_{\gamma, \gamma^{\prime}}\right) \subset \Xi_{\gamma, \gamma^{\prime}}$ E3 follows from the induction hypothesis and the construction of $\mathscr{H}_{(\Gamma, \mathscr{U})}$. Now the uniqueness. If $\mathscr{T}^{\prime}$ is another renormalization, then by E1 and E4

$$
\operatorname{supp}\left[\mathscr{T}^{\prime} \mathscr{E}_{(\Gamma, \mathscr{U})}(\underline{x})-\mathscr{T} \mathscr{E}_{(\Gamma, \mathscr{U})}(\underline{x})\right] \subset\left\{\underline{x} \in \mathbb{R}^{4 V}: x_{1}=x_{2}=\ldots=x_{V}\right\} .
$$


Hence

$$
\mathscr{T}^{\prime} \mathscr{E}_{(\Gamma, \mathcal{U})}(\underline{x})-\mathscr{T}_{(\Gamma, \mathscr{U})}(\underline{x})=D_{\Gamma} \delta\left(x_{2}-x_{1}\right) \ldots \delta\left(x_{V}-x_{V-1}\right),
$$

where $D_{\Gamma}$ is a differential operator. By E2 and E3 it is Euclidean invariant and real. Q.E.D.

\section{An Example: Analytic Renormalization}

Analytic renormalization [10] provides probably the simplest explicit renormalization scheme. We shall show that the Euclidean version of analytic renormalization is a renormalization in the sense of Sect. III. In the case of the relativistic formulation of analytic renormalization Hepp [6] proved that the corresponding set of axioms (conditions R0-R4, Sect. III) is satisfied. The main simplification of the Euclidean approach is the absence of the $\varepsilon \rightarrow 0$ problem.

Consider a distribution $\mathscr{E}_{(\Gamma, \mathscr{U})}(\underline{x}, \underline{\lambda})$ defined by (II.2) with $S(x)$ replaced by the regularized propagator $S_{\lambda, r}(x)$, which is a Fourier transform of

$$
\hat{S}_{\lambda, r}(p)=\Gamma(\lambda)^{-1} \int_{r}^{\infty} d \alpha \alpha^{\lambda-1} e^{-\lambda\left(p^{2}+\mu^{2}\right)}
$$

where $r>0, \lambda \in \mathbb{C}$ with $\operatorname{Re} \lambda>0 . \Gamma(\lambda)$ is the Euler function. Modifying slightly the argument of Speer one proves easily

Proposition. Let $\mu^{2}>0$. There is some open set $\Omega \subset \mathbb{C}^{L}$ ( $L=$ the number of propagators in (II.2)) such that $\mathscr{E}_{(\Gamma, \mathscr{U})}(\underline{x}, \underline{\lambda})=\lim _{r \rightarrow 0} \mathscr{E}_{(\Gamma, \mathscr{U})}(\underline{x}, \underline{\lambda})$ exists in $\mathscr{S}^{\prime}\left(\mathbb{R}^{4 V}\right)$, if $\underline{\lambda} \in \Omega . \mathscr{E}_{(\Gamma, \mathscr{U})}(\underline{x}, \underline{\lambda})$ is holomorphic in $\Omega$, and can be analytically continued to a function meromorphic in $\mathbb{C}^{L}$.

Define

$$
\begin{aligned}
\mathscr{T}_{A^{\mathscr{E}}(\Gamma, \mathscr{U})}(\underline{x})= & (L !)^{-1} \sum_{P \in S_{L}}(2 \pi i)^{-L} \oint_{C_{P(1)}} d \lambda_{1} \ldots \oint_{C_{P(L)}} d \lambda_{L} \\
& \cdot \mathscr{E}_{(\Gamma, \mathcal{U})}(\underline{x}, \underline{\lambda}) \prod_{j=1}^{L}\left(\lambda_{j}-1\right)^{-1},
\end{aligned}
$$

where $0<R_{1}<\ldots<R_{L}$ is any sequence satisfying the condition: $R_{i}>\sum_{j=1}^{i-1} R_{j} . C_{i}$ is the contour $|\lambda-1|=R_{i}$ oriented counterclockwise. Actually, $\mathscr{T}_{A}$ is independent of particular choice of the $R_{i}$ 's.

Theorem EA. $\mathscr{T}_{A}$ satisfies conditions E0-E4.

Proof. For the proof that $\mathscr{T}_{A}^{\mathscr{E}_{(T, \mathscr{U})}}(\underline{x}) \in \mathscr{S}^{\prime}\left(\mathbb{R}^{4 V}\right)$, see [10, p. 1407]. To see that $\mathscr{T}_{A} \mathscr{E}_{(\Gamma, \mathscr{U})}(\underline{x})$ is an extension of $\mathscr{E}_{(\Gamma, \mathscr{U})}(\underline{x})$ observe that $S\left(x_{i(\sigma)}-x_{f(\sigma)}\right)$ is a $C^{\infty}$-function, if $x_{i(\sigma)}^{0}-x_{f(\sigma)}^{0} \neq 0$. Thus, the limit $r \rightarrow 0$ can be taken trivially, and we conclude that $\mathscr{E}_{(\Gamma, \mathscr{U})}(\underline{x}, \underline{\underline{\lambda}})$ is analytic in $\mathbb{C}^{L}$. It follows from (VIII.1) that $\mathscr{T}_{A} \mathscr{E}_{(\Gamma, \mathscr{U})}(\underline{x})=\mathscr{E}_{(\Gamma, \mathscr{U})}(\underline{x})$. This proves E0. E1 follows from the fact that $\operatorname{supp}_{(I, \mathscr{U})}(\underline{x}, \underline{\lambda}) \subset \Delta^{\mathscr{U}}$. Similarly, E2 is valid, because $\mathscr{E}_{(\Gamma, \mathscr{U})}(\underline{x}, \underline{\lambda})$ is Euclidean invariant. To prove E3 observe that $\mathscr{E}_{(\Gamma, \mathscr{U})}(\underline{x}, \underline{\lambda})^{*}=\mathscr{E}_{(\Gamma, \mathscr{U})}\left(\underline{x}, \underline{\lambda}^{*}\right)$. Now we change the variables $\underline{\lambda} \rightarrow \underline{\lambda}^{*}$ in (VIII.1). This 
results in the change of the orientation of the $C_{i}$ 's, which is, however, cancelled by $(-1)^{L}$ coming from conjugation of $i^{L}$. The proof of $E 4$ is also simple. If $x \in \Xi_{\gamma, \gamma^{\prime}}$ $\left(\gamma, \gamma^{\prime}\right.$ compatible with $\left.\mathscr{U}\right)$, then

$$
\prod_{\sigma \notin \mathscr{L}(\gamma) \cup \mathscr{L}\left(\gamma^{\prime}\right)} S_{\lambda_{\sigma}}\left(x_{i(\sigma)}-x_{f(\sigma)}\right) \quad(r \rightarrow 0)
$$

is analytic in the corresponding $\lambda$ 's. Using this fact and the factorization property of $\mathscr{T}_{A}$ (property 6 of [10, p. 1407], we find:

$$
\begin{aligned}
\mathscr{T}_{A} \mathscr{E}_{(\Gamma, \mathscr{U})}(\underline{x})= & \mathscr{T}_{A} \mathscr{E}_{\left(\gamma, \mathscr{U}_{\gamma}\right)}(\underline{x}) \mathscr{T}_{A} \mathscr{E}_{\left(\gamma^{\prime}, \mathscr{U}_{\gamma^{\prime}}\right)}\left(\underline{x}_{\gamma^{\prime}}\right) \\
& \left.\cdot \mathscr{T}_{A} \prod_{\sigma \notin \mathscr{L}(\gamma) \cup \mathscr{L}\left(\gamma^{\prime}\right)} S\left(x_{i(\sigma)}-x_{f(\sigma)}\right)\right] \\
= & \mathscr{T}_{A} \mathscr{E}_{\left(\gamma, \mathscr{U}_{\gamma}\right)}\left(\underline{x}_{\gamma}\right) \mathscr{T}_{A} \mathscr{E}_{\left(\gamma^{\prime}, \mathscr{U}_{\gamma^{\prime}}\right)}\left(\underline{x}_{\gamma^{\prime}}\right) \prod_{\sigma \notin \mathscr{L}(\gamma) \cup \mathscr{L}\left(\gamma^{\prime}\right)} S\left(x_{i(\sigma)}-x_{f(\sigma)}\right) \text {. Q.E.D. }
\end{aligned}
$$

Remark. It can be shown that the BPHZ scheme [13] (and also its massless version as proved in [7]) satisfies the axioms. In particular, it follows from the equivalence theorem that the corresponding Minkowski space renormalized amplitudes exist and satisfy physical conditions. This remark shows that one can overcome the long and non-elementary argument of [8].

\section{Appendix A}

Proof of the Lemma of Sect. III

(a) The implication $\Leftarrow$ is clear. $\Rightarrow$ Due to Euclidean invariance E4 is equivalent to E4". Equation (III.4) holds on

$$
\left\{\underline{x} \in \mathbb{R}^{4 V}: x_{v}^{0} \neq x_{v^{\prime}}^{0}, v \in \mathscr{V}(\gamma), v^{\prime} \in \mathscr{V}\left(\gamma^{\prime}\right)\right\} .
$$

Given a point $x$ in (A.1) we can decompose its coordinates into $\left(\underline{x}_{\tau_{1}}\right) \cup\left(\underline{x}_{\tau_{2}}\right) \cup \ldots \cup\left(\underline{x}_{\tau_{k}}\right)$, in such a way that (i) $x_{v}^{0}<x_{v^{\prime}}^{0}$, if $v \in \mathscr{V}\left(\tau_{i}\right), v^{\prime} \in \mathscr{V}\left(\tau_{j}\right), i<j$, (ii) $\tau_{1}, \ldots, \tau_{3}, \ldots \subset \gamma, \tau_{2}, \tau_{4}, \ldots \subset \gamma^{\prime}$, or $\tau_{1}, \tau_{3}, \ldots \subset \gamma^{\prime}, \tau_{2}, \tau_{4}, \ldots \subset \gamma$. Then, by E4'

$$
\begin{aligned}
\mathscr{T} \mathscr{E}_{(\Gamma, \mathscr{U})}(\underline{x})= & \mathscr{T} \mathscr{E}_{\left(\tau_{1}, \mathscr{U}_{\tau_{1}}\right)}\left(\underline{x}_{\tau_{1}}\right) \prod_{\substack{\sigma: i(\sigma) \in \mathscr{V}\left(\tau_{1}\right) \\
f(\sigma) \in \mathscr{V}\left(\tau_{2}\right)}} S\left(x_{i(\sigma)}-x_{f(\sigma)}\right) \ldots \\
& \cdots \prod_{\substack{\sigma: i(\sigma) \mathscr{V}_{(\tau)}\left(\tau_{k}-1\right) \\
f(\sigma) \in \mathcal{V}\left(\tau_{k}\right)}} S\left(x_{i(\sigma)}-x_{f(\sigma)}\right) \mathscr{T} \mathscr{E}_{\left(\tau_{k}, \mathscr{U}_{\tau_{k}}\right)}\left(\underline{x}_{\tau_{k}}\right) \\
= & \mathscr{T} \mathscr{E}_{\left(\gamma, \mathscr{U}_{\gamma}\right)}\left(\underline{x}_{\gamma}\right) \mathscr{T} \mathscr{E}_{\left(\gamma^{\prime}, \mathscr{U}_{\gamma^{\prime}}\right)}\left(\underline{x}_{\gamma^{\prime}}\right) \prod_{\substack{\sigma: i(\sigma) \in \mathscr{V}(\gamma) \\
f(\sigma) \in \mathscr{V}\left(\gamma^{\prime}\right)}} S\left(x_{i(\sigma)}-x_{f(\sigma)}\right),
\end{aligned}
$$

where we have used the fact that $S(-x)=S(x)$. Hence $E 4^{\prime \prime}$.

(b) The proof is similar. One uses the fact that $\Delta_{+}(-x)=\Delta_{+}(x)$, if $x^{2}<0$.

\section{Appendix B}

We collect here some basic facts concerning radially analytic functions [2].

Let $A$ denote the set $\{\lambda \in \mathbb{C}:|\lambda|>0,0 \leqq \arg \lambda \leqq \pi / 2\}$, and $A^{*}=\left\{\lambda \in \mathbb{C}: \lambda^{*} \in A\right\}$. A function $f \in \mathscr{S}\left(\mathbb{R}^{4 V}\right)$ is called radially analytic in $A$ (respectively in $A^{*}$ ), if there exists a continuous mapping $(f, \lambda) \rightarrow f_{\lambda}$ from $\mathscr{S}\left(\mathbb{R}^{4 V}\right) \times A$ (respectively $\times A^{*}$ ) to $\mathscr{S}\left(\mathbb{R}^{4 V}\right)$, such that 
(i) for fixed $\underline{x} f_{\lambda}(\underline{x})$ is analytic in $\lambda \in \operatorname{Int} A$ (respectively $\operatorname{Int} A^{*}$ ).

(ii) for $\lambda>0 f_{\lambda}(\underline{x})=f\left(\lambda x_{1}^{0}, \mathbf{x}_{1} ; \ldots ; \lambda x_{V}^{0}, \mathbf{x}_{V}\right)$.

Lemma B.1. Functions radially analytic in $A$ (respectively $A^{*}$ ) exist. They form a dense subset of $\mathscr{S}\left(\mathbb{R}^{4 V}\right)$.

For the proof see the appendix of [2].

Lemma B.2. If $f \in \mathscr{S}\left(\mathbb{R}^{4 V}\right)$ is radially analytic in $A$, then for any $\alpha, D^{\alpha} f$ is also radially analytic, and

where $\left|\alpha_{0}\right|=\sum \alpha_{j}^{0}$.

$$
\left(D^{\alpha} f\right)_{i}(\underline{x})=i^{-\left|\alpha_{0}\right|} D^{\alpha} f_{i}(\underline{x})
$$

Proof. It follows from (ii) of the definition, Cauchy's integral formula, and the Lusin-Privalov theorem.

Remark. $w \in \mathscr{S}\left(\mathbb{R}^{V-1}\right)$ is said to be radially analytic, if $0<\lambda \rightarrow w_{\lambda}(\underline{\xi})=w(\underline{\lambda} \xi)$ has the required analytic continuation.

Acknowledgements. The authors want to express their gratitude to Prof. Konrad Osterwalder for proposing the problem and many helpful discussions. They are also grateful to Emil Straube for helpful discussions and his kind interest in their work.

\section{References}

1. Dyson, F.J.: The $S$ matrix in quantum electrodynamics. Phys. Rev. 75, 1736 (1949)

2. Eckmann, J.-P., Epstein, H.: Time ordered products and Schwinger functions. Commun. Math. Phys. 64, 95 (1979)

3. Epstein, H.: In: Axiomatic field theory. Chretien, M., Deser, S. (eds.). New York: Gordon and Breach 1966

4. Epstein, H., Glaser, V., Stora, R.: In: Structural analysis of multiparticle collision amplitudes in relativistic quantum field theory. Les Houches (1975)

5. Hep, K.: Proof of the Bogoliubov-Parasiuk theorem on renormalization. Commun. Math. Phys. 2, 301 (1966)

6. Hepp, K.: In: Statistical mechanics and field theory. DeWitt, C., Stora, R. (eds.). New York: Gordon and Breach 1971

7. Lowenstein, J., Zimmermann, W.: On the formulation of theories with zero-mass propagation. Nucl. Phys. B86, 77 (1975);

Lowenstein, J., Zimmermann, W.: Infrared convergence of Feynman integrals for the massless $A^{+}$ model. Commun. Math. Phys. 46, 105 (1975)

8. Lowenstein, J., Speer, E. : Distributional limits of renormalized Feynman integrals with zero-mass denominators. Commun. Math. Phys. 47, 43 (1976)

9. Osterwalder, K., Schrader, R.: Axioms for Euclidean green's functions, I and II. Commun. Math. Phys. 31, 83 (1973); 42, 281 (1975)

10. Speer, E.: Analytic renormalization. J. Math. Phys. 9, 1404 (1968)

11. Weinberg, S.: High-energy behavior in quantum field theory. Phys. Rev. 118, 838 (1960)

12. Williams, D.N.: The Euclidean loop expansion for massive $\lambda \phi_{4}^{4}$ : Through one loop. Commun. Math. Phys. 54, 193 (1977)

13. Zimmermann, W.: Convergence of Bogoliubov's method for renormalization in momentum space. Commun. Math. Phys. 15, 208 (1968)

Communicated by R. Stora

Received November 5, 1982 